\title{
A key for the determination of European species of Eosentomon Berlese, 1909 (Protura, Eosentomata, Eosentomidae)
}

\author{
Julia Shrubovych ${ }^{1,2,3}$, Ernest C. Bernard ${ }^{4}$ \\ I Institute of Soil Biology, Biology Centre Czech Academy of Sciences, Na Sádkách 7, 370 05, České Budějovice, \\ Czech Republic 2 Institute of Systematics and Evolution of Animals, Polish Academy of Sciences, ul. Stawkowska \\ 17, 31-016 Krakow, Poland 3 State Museum of Natural History, Ukrainian Academy of Sciences, Teatral'na St. \\ 18, UA 79008 L'viv, Ukraine 4 University of Tennessee, Entomology and Plant Pathology, 2505 E.J. Chapman \\ Drive, 370 Plant Biotechnology, Knoxville, TN 37996, USA \\ Corresponding author: Julia Shrubovych (shrubovych@gmail.com)
}

Academic editor: L. Deharveng | Received 30 November 2017 | Accepted 19 February 2018 | Published 12 March 2018

http://zoobank.org/4E6FA342-370E-4677-B27B-1BB8E312E139

Citation: Shrubovych J, Bernard EC (2018) A key for the determination of European species of Eosentomon Berlese, 1909 (Protura, Eosentomata, Eosentomidae). ZooKeys 742: 1-12. https://doi.org/10.3897/zookeys.742.22664

\begin{abstract}
European species of Eosentomon are examined. A taxonomic key to identification of 61 Eosentomon species is provided based on body chaetotaxy, shape, and position of sensilla on the foretarsus and shape of sensilla on the maxillary palps. Biogeographically, 13 of the known European Eosentomon species are known only from their type localities.
\end{abstract}

\section{Keywords}

Europe, Eosentomon, Protura, taxonomic key

\section{Introduction}

The proturan genus Eosentomon Berlese, 1909 has a worldwide distribution and contains approximately 310 described species (Szeptycki 2007, Bu and Yin 2007, Shrubovych and Szeptycki 2008, Nakamura and Likhitrakarn 2009, Nakamura 2010), of which 61 have been described from Europe (Szeptycki 2007, Shrubovych and Szeptycki 2008). 
A key for the identification of European Eosentomon species was created by Nosek (1973) for 14 species, in which the author differentiated species into four groups according to the shape of female squama genitalis. This approach followed Tuxen (1960), who divided worldwide Eosentomon into 11 groups based on the squama genitalis. Szeptycki $(1984,1985$ a, 1985b, 1986) wrote a series of papers with keys to identification of four groups of Polish Eosentomon species, in which cephalic chaetotaxy was used for group separation (Szeptycki, 1986). Both Nosek and Szeptycki frequently used in their keys the shape of the female squama genitalis, which made identification of males and young stages impossible except by association. The present paper contains an identification key to European Eosentomon species based primarily on chaetotaxy, shape, and position of sensilla on the foretarsus and the shape of sensilla on the maxillary palpi.

\section{Materials and methods}

Type materials were examined of 31 Eosentomon species deposited in the collection of Prof. Szeptycki in the Institute of Systematics and Evolution of Animals PAS, eight Eosentomon species in the collection of J. Rusek in the Institute of Soil Biology BCCAS and one species deposited in the collection the State Museum of Natural History NASU. Information about the taxonomy of other Eosentomon species was taken from original descriptions or redescriptions of type materials in Tuxen (1964), Nosek (1973) and various other papers. Head chaetotaxy is labelled as in Szeptycki (1984), and body chaetotaxy is labeled according to Bernard (1990).

The geographical distribution of these species is given according to recent published data (Szeptycki 2007, Shrubovych 2010, Christian 2011, Galli et al. 2011, Blesić and Mitrovski-Bogdanović 2012, Shrubovych and Sterzyńska 2015, Shrubovych et al. 2015, Shrubovych and Fiera 2016). Each species was assigned to a major biogeographic region (Alpine, Boreal, Continental, Pannonian, Mediterranean, Macaronesian) according to the European Environment Commission (2017) Natura 2000 terminology of European biogeographic regions (see map in: https:/www.eea.europa.eu/data-and-maps/figures/ biogeographical-regions-in-europe-2).

\section{Results and discussion}

Taxonomic characters used in the key are present in juvenile stages as well as the adults. The shapes of the parts of the adult female squama genitalis may have great phylogenetic value and can serve as additional characters for identification of species. The characters used in the key, such as shape of maxillary palpi, chaetotaxy of the head, shape and position of sensilla on the foretarsus and position of seta P1a on tergite VII are stable from the second juvenile stage (larva I) (Nosek 1973, Szeptycki 1965b). All setae on the notal tergites and on the abdominal segments are present from the maturus junior stage (Imadaté 1965). 
Analysis of the geographical distribution of European Eosentomon shows that nearly all species have been collected only in Europe, except for two (E. delicatum and E. mixtum) that have also been recorded from northern Africa. The majority of the species have been recorded only from Central Europe, probably due to many years of active work in this region by Josef Nosek, Josef Rusek and Andrzej Szeptycki. Sixteen species are known from Western Europe and only two species have been reported from Eastern Europe (Table 1). Nineteen species occur in Southern Europe and only two species have been noted from Northern Europe. If intensity of collection and study is correlated with number of recognized species then there are more species yet to be discovered in Europe. Thirteen of the 61 species are known only from their type localities: therefore, it is difficult to assert endemicity within such a poorly studied group of microarthropods. Nevertheless, two species could be endemics of the East Carpathians. The occurrence of E. carpaticum has been confirmed in the Carpathian Mountains of Ukraine, Romania, Hungary (Shrubovych and Sterzyńska 2015, Shrubovych et al. 2015) and in the Slovakian Carpathians (unpublished data). Therefore, it can be considered an Eastern-Carpathian endemic (Table 1). The collection of E. carpaticum in the lowlands of the Transcarpathian region is consistent with an earlier report that this species has a wide ecological plasticity and environmental distribution pattern, and can predominate in Protura communities outside of mountain habitats in azonal habitats, such as floodplain forests (Sterzyńska et al. 2012). A similar situation exists with E. enigmaticum, which was collected in the Ukrainian and Slovakian Carpathians (Shrubovych and Sterzynska 2015, unpublished data) and has been considered an Eastern-Carpathian endemic (Shrubovych and Sterzynska 2017); however, in Poland this species was found outside of mountainous habitats.

The existence of at least 61 European Eosentomon species, with only two of its species outside the continent (Mediterranean Africa), strongly suggests that the remaining continents must have many more species than are currently known from them. All of the world's Eosentomon species have ranges restricted to a single continent, and most are apparently specialized to a particular biome or specialized habitat. Much more collecting needs to be done, even in Europe, for us to understand the diversity of these enigmatic hexapods.

Table I. Distribution pattern of Eosentomon species in European biogeographical regions.

\begin{tabular}{l|c|c}
\hline \multicolumn{1}{c|}{ Species } & Countries & Biogeographical regions \\
\hline E. armatum Stach, 1927 & Nearly all of Europe & $\begin{array}{c}\text { Continental-Pannonian- } \\
\text { Mediterranean }\end{array}$ \\
\hline E. bloszyki Szeptycki, 1985 & $\begin{array}{c}\text { Poland, Czech Republic, Luxem- } \\
\text { bourg, Germany, Austria, Ukraine }\end{array}$ & Continental \\
\hline E. boedvarssoni Nosek, 1973 & Sweden & Boreal \\
\hline E. bohemicum Rusek, 1966 & Czech Republic, Poland & Continental \\
\hline E. briophillum Szeptycki, 1986 & Poland, type locality only & Continental \\
\hline E. canarinum Szeptycki, 2004 & Canary Islands & Macaronesian \\
\hline E. carolae Condé, 1947 & France, Spain & Mediterranean \\
\hline E. carpaticum Szeptycki, 1985 & Poland, Ukraine, Romania, & Alpine-Continental \\
\hline E. cetium Szeptycki \& Christian, 2000 & Austria, type locality only & Continental \\
\hline
\end{tabular}




\begin{tabular}{|c|c|c|}
\hline Species & Countries & Biogeographical regions \\
\hline E. coiffaiti Condé, 1961 & Minorca, Serbia & Macaronesian-Continental \\
\hline E. condei da Cunha, 1950 & Portugal, Spain & Mediterranean \\
\hline E. delicatum Gisin, 1945 & All Europe, North Africa & $\begin{array}{c}\text { Alpine-Boreal-Continental- } \\
\text { Pannonian-Mediterranean } \\
\end{array}$ \\
\hline E. denisi Condé, 1947 & France, Spain & Mediterranean \\
\hline E. enigmaticum Szeptycki, 1986 & $\begin{array}{c}\text { Poland, Ukraine, Romania, } \\
\text { Slovakia }\end{array}$ & Alpine-Continental \\
\hline E. fichteliense Rusek, 1988 & Germany, type locality only & Continental \\
\hline E. foliaceus Rusek, 1988 & Germany, Poland & Continental \\
\hline E. foroiuliense Torti \& Nosek, 1984 & Italy, type locality only & Continental \\
\hline E. funkei Rusek, 1988 & Germany, Luxembourg & Continental \\
\hline E. gamae Aldaba, 1986 & Portugal, type locality only & Mediterranean \\
\hline E. germanicum Prell, 1912 & Nearly all of Europe, Morocco & $\begin{array}{c}\text { Alpine-Boreal-Continental- } \\
\text { Mediterranean }\end{array}$ \\
\hline E. gisini Nosek, 1967 & Austria, Slovakia & Alpine \\
\hline E. gramineum Szeptycki, 1986 & Poland, Ukraine & Continental \\
\hline E. kamenickiense Rusek, 1974 & Czech Republic, type locality only & Continental \\
\hline E. longisquamum Szeptycki, 1986 & Poland, Austria & Continental \\
\hline E. lusitanicum Aldaba, 1986 & Portugal, type locality only & Mediterranean \\
\hline E. luxembourgense Szeptycki, 2001 & Luxembourg, Austria & Continental \\
\hline E. mariae Szeptycki, 1986 & $\begin{array}{l}\text { Poland, Austria, Germany, Lux- } \\
\text { embourg, Ukraine }\end{array}$ & Continental \\
\hline E. mirabile Szeptycki, 1984 & $\begin{array}{l}\text { Poland, Germany, Austria, Ca- } \\
\text { nary Islands, France, Ukraine }\end{array}$ & Continental-Macaronesian \\
\hline E. mixtum Condé, 1945 & $\begin{array}{c}\text { France, Austria, Czech Republic, } \\
\text { Germany, Slovakia, Madeira }\end{array}$ & $\begin{array}{c}\begin{array}{c}\text { Alpine-Continental-Maca- } \\
\text { ronesian }\end{array} \\
\end{array}$ \\
\hline E. noseki Tuxen, 1982 & Macaronesia, Spain, Italy & Macaronesian-Mediterranean \\
\hline E. occidentale Szeptycki, 1985 & Poland & Continental \\
\hline E. palustre Szeptycki \& Sławska, 2000 & Poland, type locality only & Continental \\
\hline E. parvum Szeptycki, 1986 & Austria, Poland & Continental \\
\hline E. pastorale Szeptycki, 2001 & Austria, Luxembourg & Continental \\
\hline E. paucrum Szeptycki, 2001 & Luxembourg & Continental \\
\hline E. pinetorum Szeptycki, 1984 & $\begin{array}{c}\text { Austria, Czech Republic, Poland, } \\
\text { Ukraine, Romania }\end{array}$ & Alpine-Continental \\
\hline E. pinkyae Arbea-Polite, 1990 & Spain, type locality only & Mediterranean \\
\hline E. polonicum Szeptycki, 1985 & Poland & Continental \\
\hline E. posnaniense Szeptycki, 1986 & Poland, Austria & Continental \\
\hline E. pratense Rusek, 1973 & $\begin{array}{c}\text { Czech Republic, Poland, former } \\
\text { Yugoslavia, Slovakia, Germany, } \\
\text { Austria, Ukraine }\end{array}$ & Continental \\
\hline E. rafalskii Szeptycki, 1985 & Poland, Czech Republic, Germany & Continental \\
\hline E. romanum Nosek, 1969 & Italy & Mediterranean-Continent \\
\hline $\begin{array}{l}\text { E. rusekianum Stumpp \& Szeptycki, } \\
1989\end{array}$ & Germany, Austria, Poland & Continental \\
\hline E. scytha Shrubovych \& Szeptycki, 2008 & Ukraine, type locality only & Continental \\
\hline E. semiarmatum Szeptycki, 1986 & $\begin{array}{c}\text { Balearic Islands, France, Germany, } \\
\text { Poland, Ukraine, Romania }\end{array}$ & Mediterranean-Continental \\
\hline E. sexsetosum Szeptycki, 1985 & Luxembourg, Poland & Continental \\
\hline
\end{tabular}




\begin{tabular}{l|c|c}
\hline \multicolumn{1}{c|}{ Species } & Countries & Biogeographical regions \\
\hline E. silesiacum Szeptycki, 1985 & $\begin{array}{c}\text { Germany, Czech Republic, Poland, } \\
\text { Luxembourg, Sweden }\end{array}$ & Boreal-Continental \\
\hline E. silvaticum Szeptycki, 1986 & Poland, Luxembourg, Romania & Alpine-Continental \\
\hline E. solarzi Szeptycki, 1993 & $\begin{array}{c}\text { European part of Russia, type } \\
\text { locality only }\end{array}$ & Continental \\
\hline E. stachi Rusek, 1966 & $\begin{array}{c}\text { Austria, Luxembourg, Poland, } \\
\text { Slovakia, Ukraine, Romania }\end{array}$ & Alpine- Continental \\
\hline E. stompi Szeptycki \& Weiner, 1993 & Germany, Luxembourg & Continental \\
\hline E. stumppi Rusek, 1988 & Germany, Austria & Continental \\
\hline E. sudeticum Szeptycki, 1985 & Poland, Czech Republic & Continental \\
\hline E. transitorium Berlese, 1909 & Poland & $\begin{array}{c}\text { Alpine-Boreal-Continental- } \\
\text { Pannonian-Mediterranean }\end{array}$ \\
\hline E. ulinense Szeptycki, 1999 & Luxembourg & Continental \\
\hline E. umbrosum Szeptycki, 2001 & Austria, type locality only & Continental \\
\hline E. vindobonense Szeptycki \& Christian, & Contal \\
\hline E. vulgare Szeptycki, 1984 & $\begin{array}{c}\text { Poland, Czech Republic, Germany, } \\
\text { Austria, Luxembourg, Ukraine }\end{array}$ & Continental \\
\hline E. wanda Szeptycki, 1985 & Poland, type locality only & Continental \\
\hline E. weinerae Szeptycki, 2001 & Austria, Luxembourg & Continental \\
\hline E. zodion Szeptycki, 1985 & Poland, Ukraine & Continental \\
\hline
\end{tabular}

\section{Key to the European Eosentomon species}

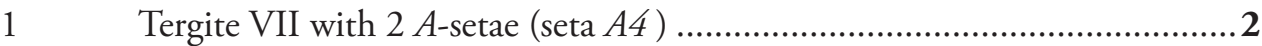

- $\quad$ Tergite VII with greater number of $A$-setae ................................................. 3

2 Tergite IV with $8 A$-setae ( $A 5$ absent), foretarsal sensillum $a$ clearly shorter than $c$ (see Rusek 1974: figs 1, 2) ...................................... E. kamenickiense

- $\quad$ Tergite IV with $10 A$-setae ( $A 5$ present), foretarsal sensilla $a$ and $c$ equal in length (see Rusek 1973: figs 1, 2, 10).......................................... E. pratense

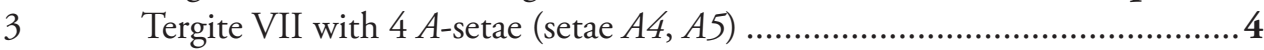

- $\quad$ Tergite VII with other number of $A$-setae................................................28

$4 \quad$ Tergite $\mathrm{V}$ with $8 A$-setae $(A 3$ absent $)$............................................................ $5^{*}$

- $\quad$ Tergite $\mathrm{V}$ with $10 A$-setae $(A 3$ present).......................................................16

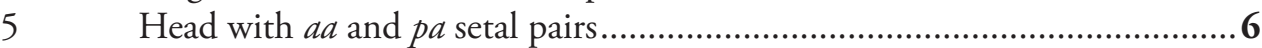

- Head with one pair or without additional setae.......................................11

Two species, E. denisi and E. condei, will key to couplet 5 but their cephalic chaetotaxy is unknown. Eosentomon denisi possesses eight $A$-setae on tergites V-VII, the female squama genitalis is of the "wheeleri" type (see Nosek 1973: 92-94, fig. $26 \mathrm{I}$ ), the foretarsus is $110 \mu \mathrm{m}$ long. The other species, E. condei, has eight $A$-setae on tergites V-VI and six $A$-setae on tergite VII, and the foretarsus length is $80 \mu \mathrm{m}$ (see Nosek 1973: 107-108). 
Seta P1a at level of P2 on tergite VII (see Szeptycki 1985a: fig. 25; Szeptycki and Sławska 2000: fig. 19)..... Seta P1a posterior to level of $P 2$, extending past hind margin of tergite VII .... 10 Foretarsal sensillum $d$ long, reaching base of t3 (Szeptycki 1985a: fig. 28; Szeptycki 2001: fig. 61) ..... Foretarsal sensillum $d$ short, reaching base of a5 (see Szeptycki 2001: fig. 81; Szeptycki and Sławska 2000: fig. 10)

Foretarsal sensillum $c$ ' long, base proximal to line $a 6-\delta 5$ (Szeptycki 1985a: fig. 27), length of foretarsus 100-105 $\mu \mathrm{m}$. E. bloszyki Foretarsal sensillum $c$ ' short, base distal to line $a 6-\delta 5$ (Szeptycki 2001: fig. 60), length of foretarsus $80-85 \mu \mathrm{m}$ Foretarsal sensillum $t 1$ nearer to $a 3^{\prime}$ than to $a 3$, rostral and subrostral setae equal in length (see Szeptycki 2001: figs 72, 85), length of foretarsus $105-115 \mu \mathrm{m}$

E. pastorale

Sensillum $t 1$ midway between $a 3$ and $a 3$ ', rostral setae shorter than subrostral setae (see Szeptycki and Sławska 2000: figs 2, 12), foretarsus 70-80 $\mu \mathrm{m}$

E. palustre

Foretarsal sensillum $c$ 'thick, proximal to line $a 6-\delta 5$ (see Nosek 1973: fig. 36B; Szeptycki 1985a: fig. 48), length of foretarsus 80-95 $\mu \mathrm{m}$ E. stachi Foretarsal sensillum $c$ ' slender, on line $a 6-\delta 5$ (see Szeptycki 1985: fig. 85), length of foretarsus 95-100 $\mu \mathrm{m}$ E. carpaticum

11 Head with $p a$ setae only, foretarsal sensillum b'2 very long, foliaceous (see Rusek 1988: figs 1D, 2B), length of foretarsus $95 \mu \mathrm{m}$ E. foliaceus Head without additional setae, foretarsal sensillum $b$ '2 shorter, sensilliform..... 12 Notal seta $P 2 a$ clearly longer than one-third the length of $P 3 a$; setae on tergite XI very short, one-sixth the length of those on tergite X (see Szeptycki 1985b: figs 48, 52, 56, 70).

Notal setae $P 2 a$ one-third the length of $P 3 a$; setae on tergite XI half as long as setae on tergite X (see Szeptycki 1985b: figs 13, 17, 22, 34)....

13 Tergite VI with $8 A$-setae ( $A 3$ absent) (see Szeptycki 1985b: fig. 39; Szeptycki and Weiner 1993: fig. 12)

Tergite VI with 6 A-setae (A1, $A 3$ absent) (see Szeptycki 1985b: fig. 57)

E. sexsetosum

14 Dorsal sensillum on maxillary palpus longer than lateral sensillum, rostral and subrostral setae subequal (see Szeptycki 1985b: fig. 46), length of foretarsus $100-110 \mu \mathrm{m}$

E. occidentale

- Sensilla on maxillary palpus equal in length, rostral and subrostral setae subequal (see Szeptycki and Weiner 1993: fig. 5), length of foretarsus 85-100 $\mu \mathrm{m}$

E. stompi

15 Tergite IV with $8 A$-setae ( $A 3$ absent), dorsal sensillum on maxillary palpus longer than lateral sensillum (see Rusek 1966: fig. 7; Szeptycki 1985b: figs 4, 6) E. bohemicum

- $\quad$ Tergite IV with $10 A$-setae, sensilla on maxillary palpus equal in length (see Szeptycki 1985: figs 23, 30).

E. polonicum 


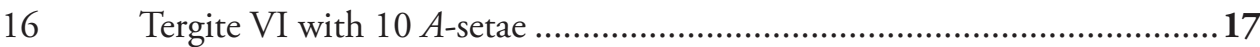

Tergite VI with 8 -setae ......................................................................18

17 Foretarsal sensilla $a$ and $c$ short, sensillum $f 2$ very short, one-fifth length of $f 1$ (see Torti and Nosek 1984: fig. 1B), length of foretarsus $112 \mu \mathrm{m}$......E. foroiuliense Foretarsal sensillum $a$ longer than $c$, sensilla $f 1$ and $f 2$ nearly equal in length, female squama genitalis with small beak-like terminus (see Arbea-Polite 1990: figs $2 \mathrm{a}, 11$ ), length of foretarsus $75-85 \mu \mathrm{m}$ E. pinkyae

18 Seta $P 1 a$ at level of $P 2$ on tergite VII (see Szeptycki 2001: figs 19, 20)...... 19 Seta $P 1 a$ posterior to level of $P 2$ on tergite VII ......................................21

19 Foretarsal sensilla $a$ and $c$ equal in length, female squama genitalis of "romanum" type (see Nosek 1973: figs 38A, J, J')

E. romanum Foretarsal sensillum $a$ clearly shorter than $c$, female squama genitalis of "transitorium" type (see Szeptycki 2001: fig. 17; Nosek 1973: fig. 33 I)

Foretarsal sensillum $c$ proximal to base of $a 6$ or to line $a 6-\delta 5$, broad (see Szeptycki 2001: figs 4, 12, 14), length of foretarsus 65-75 $\mu \mathrm{m}$.... E. luxembourgense Foretarsal sensillum $c$ to line $a 6-\delta 5$, slender (see Nosek 1973: fig. 33 A), length of foretarsus $90-100 \mu \mathrm{m}$ E. delicatum Foretarsal sensillum $c^{\prime}$ on line $a 6-\delta 5$ 22

Foretarsal sensillum $c$ proximal to line $a 6-\delta 5 \ldots \ldots \ldots \ldots \ldots \ldots \ldots \ldots \ldots \ldots \ldots \ldots \ldots \ldots . . .24$

22 Foretarsal sensillum $t 1$ nearer to $a 3^{\prime}$ than to $a 3$, sensilla on maxillary palpus nearly equal in length (see Szeptycki 1985a: figs 102, 109) E. wanda Foretarsal sensillum $t 1$ midway between $a 3$ and $a 3$ ' or nearer to $a 3$, dorsal sensillum on maxillary palpus clearly longer than lateral (see Szeptycki 1985a: figs 122, 125, Shrubovych and Szeptycki 2008: figs 5, 20).... Sensillum $t 1$ midway between $a 3$ and $a 3$, rostral and subrostral setae equal in length (Shrubovych and Szeptycki 2008: figs 4, 9) E. scytha Sensillum $t 1$ much closer to $a 3$ than to $a 3^{\prime}$, rostral setae slightly shorter than subrostral setae (see Szeptycki 1985a: figs 120, 125) E. zodion Head with $a a$ and $p a$ setae 25 Head with pa setae only (see Szeptycki 2001: fig. 26) 27

25 Sensilla on maxillary palpus thick, foretarsal sensillum $a$ half the length of $c$ (see Szeptycki 2004: figs 48, 51) 26 Sensilla on maxillary palps slender, foretarsal sensilla $a$ and $c$ nearly equal in length (see Szeptycki 1985a: figs 66, 71) E. armatum

26 Dorsal sensillum on maxillary palpus clearly longer than lateral sensillum (see Szeptycki 2004: fig. 48), length of foretarsus 95-100 $\mu \mathrm{m}$

E. noseki

Sensilla on maxillary palpus nearly equal in length (see Szeptycki 2004: fig. 26), length of foretarsus $70-80 \mu \mathrm{m}$ E. canarinum

Foretarsal sensillum $c^{\prime}$ in half distance between $a 6-\delta 4$, seta $P 2 a$ on nota equal in length to P3a (see Szeptycki: figs 32, 37), length of foretarsus $65 \mu \mathrm{m}$

E. umbrosum

- $\quad$ Foretarsal sensillum $c^{\prime}$ closer to $\delta 4^{\prime}$ than to $a 6$, seta $P 2 a$ shorter than P3a (see Rusek 1988: fig. 3B), length of foretarsus $75-85 \mu \mathrm{m}$ E. stumppi 
29 Tergite VII with $8 A$-setae ( $A 3$ absent) (see Aldaba 1986: fig. 17)..............30

30 Sternites IX - X with 6 setae, female squama genitalis of "wheeleri" type (Nosek 1973: p. 95; Tuxen 1964: fig. 105)

E. carolae

Sternites IX - X with 4 setae, female squama genitalis of "transitorium" type (see Aldaba 1986: table 2, fig. 18)

E. gamae

Tergite VI with $10 A$-setae

Tergite VI with $8 A$-setae

Sternites IX - X with 6 setae (sternite X with 4 setae in maturus junior)......48

Head with $a a$ and $p a$ setae (J. Rusek, pers. comm.; Arbea-Polite 1990: fig. 15a), seta Pla passing hind margin of tergite VII (see Nosek 1973: fig. 37H; Arbea-Polite 1990: fig. 7)....

Foretarsal sensillum $t 1$ midway between $a 3$ and a3', rostral seta evidently shorter than subrostral (see Nosek 1973: fig. 37B, C), length of foretarsus $86 \mu \mathrm{m}$

Foretarsal sensillum $t 1$ near to $a 3$ ', rostral and subrostral setae equal in length (see Arbea-Polite 1990: fig. 2a, 24), length of foretarsus 77-86 $\mu \mathrm{m}$......

Head without additional setae

E. pinkyae

Head with $p a$ setae.

Basal seta $D 2$ on hind leg about half the length of D1 (see Szeptycki 1985b: fig. 85)

Basal seta D2 on hind leg subequal with D1 (see Szeptycki 1985b: figs 108, 109).

Basal seta $D 2$ on hind leg spine-like

39 Sensilla on maxillary palps short and equal in length (see Szeptycki 1986: fig. 73, Szeptycki and Christian 2000: fig. 3)

40 Notal setae $P 1$ longer than $P 1$, foretarsal sensillum $f 1$ spatuliform (see Szeptycki 1986a: figs 74, 81)

Notal setae $P 1$ shorter than P1a, foretarsal sensillum $f 1$ filiform (see Szeptycki and Christian 2000: figs 4, 10) E. vindobonense Length ratio of notal setae P1:P1a $\geq 1.5$ (see Szeptycki 1986a: fig. 31) 
42 Sensilla on maxillary palpus nearly equal in length (see Szeptycki 1986: fig. 158; Szeptycki and Christian 2000: fig. 24) ........................................43 Lateral sensillum of maxillary palpus much longer than dorsal sensillum (see Szeptycki 1986a: figs 107, 123, 138)

43 Notal setae $P 1 a$ and $P 1$ nearly equal in length (see Szeptycki 1986a: fig. 152), length of foretarsus $85-90 \mu \mathrm{m}$

E. longisquamum

Notal seta P1 a shorter than P1 (Szeptycki and Christian 2000: fig. 28), length of foretarsus $100-115 \mu \mathrm{m}$

E. cetium

Seta $P 1 a$ on tergites I - VI longer than $P 1$, foretarsal sensillum $t 1$ nearer to $a 3$ than to $a 3^{\prime}$, sensillum $t 3$ longer than $c$ ', length of foretarsus less than $100 \mu \mathrm{m}$

Seta $P 1 a$ on tergites I - VI equal in length or shorter than $P 1$, foretarsal sensillum $t 1$ midway between $a 3$ and $a 3^{\prime}$ or slightly closer to $a 3$ ', sensillum $t 3$ short, equal in length to $c$, length of foretarsus 100-110 $\mu$ m (see Szeptycki 1986a: figs 115, 116)

E. silvaticum

Tracheal camerae long, slender; foretarsal sensillum $d$ long, reaching base of $a 6$, length of foretarsus 90-100 $\mu \mathrm{m}$ (see Szeptycki 1986a: figs 125, 126, $130,131)$

E. semiarmatum

- $\quad$ Tracheal camerae short, stocky; foretarsal sensillum $d$ short, not reaching base of $a$, length of foretarsus 75-85 $\mu \mathrm{m}$ (see Szeptycki 1986a: figs 140, 145) ....

E. parvum

Seta $P 1 a$ at level $P 2$ on tergite VII

Seta $P 1 a$ slightly posterior to $P 2$ and extending past hind margin of tergite VII (see Szeptycki 1986a: fig. 63).

E. posnaniense

47 Rostral seta thinner than subrostral seta (see Szeptycki 1986a: fig. 6), lateral sensillum on maxillary palpus longer than dorsal sensillum (see Nosek 1973: fig. 28C'; Szeptycki 1986a: fig. 7), foretarsal sensillum $t 1$ nearer to $a 3$ than to a3'(see Nosek 1973: fig. 28A; Szeptycki 1986a: fig. 20) or midway between a3 and $a 3$ ' (see Szeptycki 1986a: fig. 21)....

E. transitorium

- $\quad$ Rostral seta thicker than subrostral seta, sensilla on maxillary palpus nearly equal, foretarsal sensillum $t 1$ nearer $a 3$ ' than a3 (see Szeptycki 1986a: figs 86,100$)$

E. mariae

$48 \quad$ Seta $P 1 a$ at level of $P 2$ on tergite VII

Seta $P 1$ a posterior to level of $P 2$ on tergite VII

49 Foretarsal sensillum $f 1$ thickened apically, body length more than $1600 \mu \mathrm{m}$ (see Nosek 1973: fig. 32A; Rusek 1988: p. 229) E. mixtum

- $\quad$ Foretarsal sensillum $f 1$ not thickened apically, body length less than $1600 \mu \mathrm{m}$

50 Foretarsal sensillum $f 1$ thick, thicker than sensillum a (see Aldaba 1986: fig. 5), body length less than $850 \mu \mathrm{m}$ E. lusitanicum

- $\quad$ Foretarsal sensillum $f 1$ thin (see Rusek 1988: fig. 8A), body length more than $1300 \mu \mathrm{m}$ 
51 Foretarsal sensillum $t 1$ midway between $a 3$ and $a 3$ ', sensillum $f 2$ sensilliform (see Rusek 1988: fig. 8A, B), body length about $1460 \mu \mathrm{m}$ E. funkei

- $\quad$ Foretarsal sensillum $t 1$ nearer $a 3$ than $a 3$ ', sensillum $f 2$ spatuliform (see Nosek 1973: fig. 35A), body length about $1300 \mu \mathrm{m}$ E. coiffaiti

52 Head with both $a a$ and $p a$ setae 53 Head with aa or pa setae 54

53 Foretarsal sensillum $a$ longer than half the length of $c$, sensillum $f 1$ spatulate (see Szeptycki 1984: fig. 38).

E. mirabile

- $\quad$ Foretarsal sensillum $a$ shorter than half the length of $c$, sensillum $f 1$ filiform (see Szeptycki 2001: figs 103, 105)

E. weinerae

54 Head with aa setae (see Stumpp and Szeptycki 1989: fig. 8) . E. rusekianum

- Head with pa setae only 55

55 Lateral sensillum on maxillary palpus clearly longer than dorsal, spatulate dilation on foretarsal sensilla $e$ and $g$ short, about third of sensillum length, tracheal camerae long (see Szeptycki 1984: figs 7, 9, 11, 12) E. vulgare

- $\quad$ Sensilla on maxillary palps nearly equal in length, spatulate dilation on foretarsal sensilla $e$ and $g$ long, about half of sensillum length, tracheal camerae short and thickened (see Rusek 1988: fig. 6D; Szeptycki 1984: figs 20, 26, 27) 56

56 Foretarsal sensillum $a$ about half of $c$ length, lateral sensillum on maxillary palpus slightly shorter than dorsal (see Szeptycki 1986a: figs 20, 26)

E. pinetorum

- $\quad$ Foretarsal sensillum $a$ about equal in length to $c$, sensilla on maxillary palpus equal in length (see Rusek 1988: fig. 5A, 6D)

E. fichteliense

57 Sternites IX - X with 4 setae

58

Sternites IX - X with 6 setae, head with pa setae (see Nosek 1973: fig. 32F'; Szeptycki 1984: p. 200)

E. germanicum

58 Head without additional setae (Szeptycki 1985b: p. 532), length of foretarsus $100-110 \mu \mathrm{m}$ E. sudeticum

- $\quad$ Head with $\quad$ pa setae, length of foretarsus $\leq 85 \mu \mathrm{m}$ 59

59 Head with both $a a$ and pa setae (see Szeptycki 1999: fig. 1), length of foretarsus $80-85 \mu \mathrm{m}$

E. ulinense

- $\quad$ Head with only pa setae (see Szeptycki 1993: fig. 43), length of foretarsus 70-80 $\mu \mathrm{m}$

E. solarzi

\section{Acknowledgements}

The authors are grateful to Prof. Josef Rusek for his valuable comments on taxonomy and to Jakub Sternalski for his help with preparation this manuscript. We also thank the peer reviewers, Loris Galli and Yun Bu, and subject editor Louis Deharveng for their valuable remarks and corrections of the text. 


\section{References}

Aldaba J (1986) Descripción de dos nuevas especies del género Eosentomon Berlese (Protura: Insecta) de Portugal. Actas VIII Jornadas de la Asociación española de Entomología, Sevilla, 203-212.

Arbea-Polite JI (1990) Eosentomon pinkyae n. sp. (Protura: Eosentomidae) de Zaragoza (España). Eos 66: 15-24.

Bernard EC (1990) New species, clarifications, and changes in status within Eosentomon Berlese (Hexapoda: Protura: Eosentomidae) from the United States. Proceedings of the Biological Society of Washington 103: 861-890.

Blesić B, Mitrovski-Bogdanović A (2012) Protura in Serbia. Kragujevac Journal of Science 34: 101-106. http:/www.pmf.kg.ac.rs/kjs/images/volumes/vol34/kjs34blesicmitriovski101.pdf

Bu Y, Yin WY (2007) The Protura from Xinjiang, Northwestern China. Zootaxa 1437: 29-46. Christian E (2011) Protura (Insecta). In: Schuster R (Ed.) Checklisten der Fauna Osterreichs, No. 5 Protura (Insecta), Opiliones (Arachnida), Pseudoscorpiones (Arachnida), Tipulidae (Insecta: Diptera). Biosystematics and Ecology Series. Volume 28. Austrian Academy of Sciences Press, Wienna, 1-9.

European Environment Commission (2017) The biogeographical regions. http://ec.europa.eu/ environment/nature/natura2000/sites_hab/biogeog_regions/index_en.htm.

Galli L, Capurro M, Torti K (2011). Protura of Italy, with a key to species and their distribution. ZooKeys 146: 19-67. https://doi.org/10.3897/zookeys.146.1885

Nakamura O (2010) Taxonomic revision of the family Eosentomidae (Hexapoda: Protura) from Japan. Zootaxa 2701: 1-109. http://mapress.com/zootaxa/2010/f/z02701p109f.pdf

Nakamura O, Likhitrakarn N (2009) Protura (Hexapoda) from Doi Suthep-Pui National Park, Chiang Mai, Thailand. Zootaxa 2121: 1-16.

Nosek J (1973) The European Protura. Their taxonomy, ecology and distribution with keys for determination. Muséum D’Histoire Naturelle, Genève, 345 pp.

Rusek J (1966) Einige neue und interessante Proturen- und Dipluren-Arten aus der Tschechoslowakei (Apterygota). Acta entomologica bohemoslovaca 63: 348-372.

Rusek J (1973) Eosentomon pratensis sp. n. (Protura) aus Süd-Mähren. Acta entomológica bohemoslovaca 70: 55-59.

Rusek J (1974) Eosentomon kamenickiense sp. n. (Protura) aus Ost-Bohmen. Acta entomológica bohemoslovaca 71: 342-345.

Rusek J (1988) New Eosentomon and Acerentulus species (Protura) from Federal Republic Germany. Věsnic Československé Společnosti zoologické 52: 217-237.

Shrubovych J (2010) Taxonomical richness and chorological structure of proturan fauna (Protura) in Ukraine. Science Bulletin of the Uzhgorod University (Seria Biologia) 29: 75-81. [In Ukrainian with English summary]

Shrubovych J, Fiera C (2016) New records of Protura (Entognatha, Arthropoda) from Romania, with an identification key to Romanian species. ZooKeys 552: 33-48. https://doi.org/10.3897/ zookeys. 552.6613

Shrubovych J, Fiera C, Pfliegler W (2015) Protura of Bükk Mountain (Hungarian Carpathians). In: Studeniak IP, Roshko VG, Chumak VO, Mirutenko VV (Eds) Uzhhorod entomologi- 
cal readings $-2015.15^{\text {th }}$ International Conference, Uzhorod (Ukraine), September, 2015. Uzhorod National University, Uzhorod, 15 pp.

Shrubovych J, Sterzyńska M (2015) The fauna of Protura in Ukrainian Carpathians. In: Studeniak IP, Roshko VG, Chumak VO, Mirutenko VV (Eds) Uzhhorod entomological readings $-2015.15^{\text {th }}$ International Conference, Uzhorod (Ukraine), September, 2015. Uzhorod National University, Uzhorod, 16 pp.

Shrubovych J, Sterzyńska M (2017) Diversity and distributional pattern of soil microarthropods (Protura) across a transitional zone in Ukraine. The Canadian Entomologist 149: 628-638. https://doi.org/10.4039/tce.2017.30

Sterzyńska M, Orlov O, Shrubovych J (2012) Effect of hydrologic disturbance regimes on Protura variability in a river floodplain. Annales Zoologici Fennici 49: 309-320. https://doi.org/10.5735/086.049.0504

Shrubovych J, Szeptycki A (2008) Eosentomon scytha n. sp. a new species from Southern Ukraine (Protura: Eosentomidae). Genus 19(1): 1-6.

Stumpp J, Szeptycki A (1989) Eosentomon rusekianum, sp. n., a new species of Protura (Arthropoda: Insecta) from South Germany. Carolinea 47: 141-146.

Szeptycki A (1984) Three new species of Eosentomon Berlese, 1909 from Poland with redescription of Eosentomon germanicum Prell, 1912 (Protura). Polskie Pismo entomologiczne 54: 195-213.

Szeptycki A (1985a) Polish Protura. II. Eosentomon delicatum Gisin, 1945 and related species.

Polskie Pismo entomologiczne 55: 139-186.

Szeptycki A (1985b) Polish Protura. III. Eosentomon bohemicum Rusek, 1966 and related species. Polskie Pismo entomologiczne 55: 531-574.

Szeptycki A (1986a) Polish Protura. IV. Eosentomon "transitorium” group. Polskie Pismo entomologiczne 56: 481-530.

Szeptycki A (1986b) Remarks on the prelarva and postembryonic development of Protura. In:

Dallai R (Ed.) 2 $2^{\text {nd }}$ International Seminar on Apterygota, University of Siena, Siena, 243-248.

Szeptycki A (1993) Three new Protura from Western Caucasus. Acta zoologica cracoviensia 36: 29-43.

Szeptycki A (1999) Eosentomon ulinense sp. n. from Poland (Protura: Eosentomidae). Polskie Pismo entomologiczne 68: 211-216.

Szeptycki A (2001) New Eosentomon species from Luxembourg (Protura: Eosentomidae).

Genus 12: 237-267.

Szeptycki A (2004) Protura of the Canary Islands (Arthropoda: Protura). Genus 15: 301-322.

Szeptycki A (2007) Catalogue of the world Protura. Acta zoologica cracoviensia 50B: 1-210.

Szeptycki A, Sławska M (2000) Eosentomon palustre sp. n. from Northern Poland (Protura:

Eosentomidae). Genus 11(2): 105-111.

Szeptycki A, Christian E (2000) Two new Eosentomon species from Austria (Insecta: Protura:

Eosentomidae). Annalen des naturhistorischen Museums in Wien 102: 83-92.

Szeptycki A, Weiner WM (1993) Eosentomon stompi sp. n., a new Protura from Luxembourg. Acta zoologica cracoviensia 35: 413-421.

Tuxen SL (1964) The Protura. A revision of the species of the world with keys for determination. Hermann, Paris, 360 pp. 